\title{
Functional overexpression of genes involved in erythritol synthesis in the yeast Yarrowia lipolytica
}

\author{
Aleksandra M. Mirończuk ( 1 , Anna Biegalska and Adam Dobrowolski
}

\begin{abstract}
Background: Erythritol, a four-carbon polyol synthesized by microorganisms as an osmoprotectant, is a natural sweetener produced on an industrial scale for decades. Despite the fact that the yeast Yarrowia lipolytica has been reported since the 1970s as an erythritol producer, the metabolic pathway of this polyol has never been characterized. It was shown that erythritol synthesis in yeast occurs via the pentose phosphate pathway (PPP). The oleaginous yeast Y. lipolytica is a good host for converting inexpensive glycerol into a value-added product such as erythritol. Glycerol is a renewable feedstock which is produced on a large scale as a waste product by many branches of industry.

Results: In this study, we functionally overexpressed four genes involved in the pentose phosphate pathway (PPP): gene YALIOE06479g encoding transketolase (TKL1), gene YALIOF15587g encoding transaldolase (TAL1), gene YALIOE22649g encoding glucose-6-phosphate dehydrogenase (ZWF1), and gene YALIOB15598g encoding 6-phosphogluconate dehydrogenase (GND1). Here, we show that the crucial gene for erythritol synthesis in Y. lipolytica is transketolase. Overexpression of this gene results in a twofold improvement in erythritol synthesis during a shakeflask experiment $(58 \mathrm{~g} / \mathrm{L}$ ). Moreover, overexpression of TKL 1 allows for efficient production of erythritol independently from the supplied dissolved oxygen. Fermentation conducted in a 5-L bioreactor at low agitation results in almost $70 \%$ higher titer of erythritol over the control strain.

Conclusion: This work presents the importance of the PPP in erythritol synthesis and the feasibility for economic production of erythritol from glycerol by the yeast Y. lipolytica.
\end{abstract}

Keywords: Yarrowia lipolytica, Erythritol, Glycerol, Transketolase, Pentose phosphate pathway

\section{Background}

Erythritol is a well-known natural sweetener, commonly occurring in fruits, wine, and honey [1]. This four-carbon compound belongs to the group of polyols, the socalled sweet alcohols. Erythritol does not change the insulin level in blood; thus, it can be used by diabetics. Due to its impressive properties such as low energy value $(\sim 0.2 \mathrm{kcal} / \mathrm{g})$, natural sweet taste $(70 \%$ of sucrose sweetness), and the highest no-effect dose for causing diarrhea among the polyols [2], erythritol has been produced for decades. On the industrial scale, erythritol is produced in

\footnotetext{
*Correspondence: aleksandra.mironczuk@upwr.edu.pl

Department of Biotechnology and Food Microbiology, Wroclaw

University of Environmental and Life Sciences, Chełmońskiego 37,

51-630 Wrocław, Poland
}

aerobic fermentation processes using Torula sp. [3] and Moniliella sp. [4]. Moreover, other microorganisms such as the yeast Yarrowia lipolytica are also able to produce erythritol [5].

Erythritol is produced as an osmoprotectant by osmophilic yeast and some bacteria as a cell response to high osmotic pressure of the environment. This feature is used in industry, where highly osmotic media (up to $40 \%$ of glucose) are applied to stimulate erythritol synthesis.

Many studies have been performed to obtain highproduction organisms and to improve fermentation conditions for high productivity [6-9]. However, the most important issue in polyol synthesis is the high-production costs (caused by expensive media), oxygen supply and a high content of byproducts such as glycerol, 
ribitol, mannitol, and organic acids. For economic reasons, an alternative, low-value carbon source for erythritol biosynthesis is sought. Good substitutes of glucose for industrial applications are agricultural wastes, biodiesel byproducts, or other renewable feedstocks such as glycerol. Every year, crude glycerol is produced in huge quantities by many industrial branches, e.g., in biodiesel production, fat saponification, stearin production, and alcoholic beverage production units [10]. Despite the fact that crude glycerol is contaminated by many undesired compounds such as methanol, salts, and matter organic non-glycerol (MONG), some microorganisms are capable of metabolizing it; one of them is Y. lipolytica [11]. The unconventional yeast $Y$. lipolytica is a well-studied oleaginous model organism for investigating lipid production and accumulation in eukaryotic cells. However, it also possesses capability for production of polyols and organic acid [12-18]. Despite many studies on erythritol synthesis using $Y$. lipolytica, the metabolic pathway of this phenomenon has never been described. It was shown that in fungus and yeast, erythritol synthesis occurs via the pentose phosphate pathway (PPP) $[19,20]$ and in the final step, erythrose reductase (ER) reduces erythrose to erythritol with concomitant $\mathrm{NAD}(\mathrm{P}) \mathrm{H}$ oxidation [1].
The aim of this study was to identify the key genes involved in erythritol synthesis. In this study, we overexpressed genes involved in PPP, transketolase (TKL1, YALIOE06479g), transaldolase (TAL1, YALIOF15587g), and two dehydrogenases: ZWF1 (YALIOE22649g) and GND1 (YALIOB15598g), responsible for production of reducing agents in the cell, to verify their roles in erythritol biosynthesis. Moreover, we found that the engineered strains are able to produce erythritol at low aeration, this being beneficial for downstream processing. The gene which has the highest impact on erythritol synthesis is transketolase.

\section{Methods}

\section{Microorganisms, media, and culture conditions}

The $Y$. lipolytica strains used in this study were derived from the strain Y. lipolytica MK1 [21]. All the strains used in this study are listed in Table 1 . In Table 2, the gene name, gene symbol, and abbreviation are summarized.

Escherichia coli strains were cultivated in LB medium according to standard protocols. Rich yeast extract peptone glucose (YPD) medium was used for the yeast inoculum preparation and contained $1 \%(\mathrm{w} / \mathrm{v})$ yeast extract, $1 \%(\mathrm{w} / \mathrm{v})$ peptone, and $2 \%(\mathrm{w} / \mathrm{v})$ glucose.

Table 1 Strains and plasmids used in this study

\begin{tabular}{|c|c|c|}
\hline Strain & Genotype or plasmid & Source \\
\hline \multicolumn{3}{|l|}{ E. coli } \\
\hline $\mathrm{DH} 5 \mathrm{a}$ & 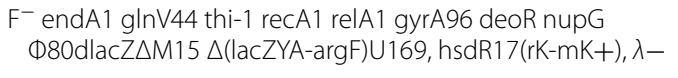 & {$[33]$} \\
\hline $\mathrm{DH} 5 \mathrm{a}$ & $\mathrm{pAD}, \mathrm{UAS1}_{\mathrm{B} 16} \mathrm{TEF}$ promoter & This study \\
\hline $\mathrm{DH} 5 \mathrm{a}$ & pAD-TKL1, YALIOE06479g & This study \\
\hline $\mathrm{DH} 5 \mathrm{a}$ & pAD-TAL1, YALIOF15587g & This study \\
\hline $\mathrm{DH} 5 \mathrm{a}$ & pAD-GDN1, YALIOB15598g & This study \\
\hline $\mathrm{DH} 5 \mathrm{a}$ & pAD-ZWF1, YALIOE22649g & This study \\
\hline Dh5 a & phrGFP I-C & Agilent \\
\hline Dh5 a & pAD-hrGFP & This study \\
\hline Dh5 a & $P_{T K L 1}-g f p$ & This study \\
\hline Dh5 a & $P_{\text {TAL1 }}-g f p$ & This study \\
\hline Dh5 a & $P_{G D N 1}-g f p$ & This study \\
\hline Dh5 a & $P_{Z W F 1}-g f p$ & This study \\
\hline \multicolumn{3}{|l|}{ Y. lipolytica } \\
\hline AMM & MATA, MK1: ura3-302 & {$[28]$} \\
\hline MK1 & MATA, UV-mutant & {$[21]$} \\
\hline AMM pAD-TKL1 & MATA, MK1: ura3-302, overexpression YALIOE06479g & This study \\
\hline AMM pAD-TAL1 & MATA, MK1: ura3-302, overexpression YALIOF15587g & This study \\
\hline AMM PAD-GDN1 & MATA, MK1: ura3-302, overexpression YALIOB15598g & This study \\
\hline AMM pAD-ZWF1 & MATA, MK1: ura3-302, overexpression YALIOE22649g & This study \\
\hline AMM $P_{T K L 1}-g f p$ & MATA, MK1: ura3-302, promoter TKL1 fused to hrgfp & This study \\
\hline AMM $P_{T A L 1}-g f p$ & MATA, MK1: ura3-302, promoter TAL1 fused to hrgfp & This study \\
\hline AMM $P_{G D N 1}-g f p$ & MATA, MK1: ura3-302, promoter GDN1 fused to hrgfp & This study \\
\hline$A M M P_{Z W F}-g f p$ & MATA, MK1: ura3-302, promoter ZWF1 fused to hrgfp & This study \\
\hline
\end{tabular}


Table 2 Description of genes overexpressed in Y. lipolytica

\begin{tabular}{lll}
\hline Gene & Number & Function \\
\hline TKL1 & YALIOE06479g & Transketolase \\
TAL1 & YALIOF15587g & Transaldolase \\
ZWF1 & YALIOE22649g & Glucose-6-phosphate dehydrogenase \\
GND1 & YALIOB15598g & 6-Phosphogluconate dehydrogenase \\
\hline
\end{tabular}

During shake-flask experiments, the cultures were grown in 0.3-L Erlenmeyer flasks or 0.25-L baffled flasks containing $0.03 \mathrm{~L}$ medium on a rotary shaker (CERTOMAT IS, Sartorius Stedim Biotech) at $28{ }^{\circ} \mathrm{C}$ at $280 \mathrm{rpm}$. Erythritol synthesis was conducted in Erythritol Synthesis Medium (g/L): 100 glycerol (Chempur, Poland), 2.3 $\left(\mathrm{NH}_{4}\right)_{2} \mathrm{SO}_{4}$ (Chempur), $1 \mathrm{MgSO}_{4} \times 7 \mathrm{H}_{2} \mathrm{O}$ (Chempur), $0.23 \mathrm{KH}_{2} \mathrm{PO}_{4}$ (Chempur), $\mathrm{NaCl} 26.4$ (Chempur), 1 yeast extract (Merck, Germany), and $3 \mathrm{CaCO}_{3}, \mathrm{pH}$ 3.0.

The Control Medium was as follows: (g/L): 100 glucose (Merck, Germany), $2.3\left(\mathrm{NH}_{4}\right)_{2} \mathrm{SO}_{4}$ (Chempur), 1 $\mathrm{MgSO}_{4} \times 7 \mathrm{H}_{2} \mathrm{O}$ (Chempur), $0.23 \mathrm{KH}_{2} \mathrm{PO}_{4}$ (Chempur), $\mathrm{NaCl} 26.4$ (Chempur), 1 yeast extract (Merck), and 3 $\mathrm{CaCO}_{3}, \mathrm{pH} 3.0$.

\section{Bioscreen C}

The yeast strain was grown in 100-well plates in $200 \mu \mathrm{L}$ of YNB supplemented with glycerol $2 \%(\mathrm{w} / \mathrm{v})$ or glucose $2 \%(\mathrm{w} / \mathrm{v})$. First, the strains were grown for $24 \mathrm{~h}$ in YPD medium containing $1 \%(\mathrm{w} / \mathrm{v})$ yeast extract, $1 \%(\mathrm{w} / \mathrm{v})$ peptone, and $2 \%(\mathrm{w} / \mathrm{v})$ glucose. The cells were washed and inoculated to an $\mathrm{OD}_{600}$ of 0.15 in each well. Quintuple experiments were performed at $28^{\circ} \mathrm{C}$ under constant agitation with a Bioscreen C (Oy Growth Curves Ab Ltd., Finland). Growth was monitored by measuring the optical density at 420-560 $\mathrm{nm}$ every $30 \mathrm{~min}$ for $72 \mathrm{~h}$.

\section{Bioreactor studies}

To prepare an inoculation culture for fermentation in a bioreactor, the cultures were grown in 0.3-L Erlenmeyer flasks (containing $0.1 \mathrm{~L}$ of YPD medium) on a shaker at $28{ }^{\circ} \mathrm{C}$ for $72 \mathrm{~h}$ at $140 \mathrm{rpm}$. Erythritol production was conducted in a medium consisting of $(\mathrm{g} / \mathrm{L}): 150$ glycerol, 2.3 $\left(\mathrm{NH}_{4}\right)_{2} \mathrm{SO}_{4}, 1 \mathrm{MgSO}_{4} \times 7 \mathrm{H}_{2} \mathrm{O}, 0.23 \mathrm{KH}_{2} \mathrm{PO}_{4}, \mathrm{NaCl} 26.4$, and 1 yeast extract, $\mathrm{pH}$ 3.0. An inoculum of $0.2 \mathrm{~L}$ was introduced into the bioreactor containing the production medium. The cultivations were performed in a $5-\mathrm{L}$ jar bioreactor (Biostat B Plus, Sartorius, Germany) with a working volume of $2 \mathrm{~L}$ at $28{ }^{\circ} \mathrm{C}$. The aeration was fixed at $0.8 \mathrm{~L} / \mathrm{min}$. The stirrer speed was adjusted to $500 \mathrm{rpm}$. The $\mathrm{pH}$ was maintained automatically at 3.0 via the addition of $\mathrm{NaOH}(40 \% \mathrm{w} / \mathrm{v})$. The amount of the supplied $\mathrm{NaOH}$ has been taken into account during calculations of the metabolite concentrations. In order to limit evaporation during the batch cultures, the exhaust gases were passed into the exhaust condenser in which the moisture was removed and returned to the vessel. The cultures were performed in three replicates.

\section{Cloning and transformation protocols}

All restriction enzymes were purchased from FastDigest Thermo Scientific (USA), and all of the digestions were performed according to standard protocols. The PCR reactions were set up using recommended conditions and Phusion high-fidelity DNA polymerase (Thermo Scientific). The ligation reactions were performed for $10 \mathrm{~min}$ at room temperature using T4 DNA Ligase (Thermo Scientific). The gel extractions were performed using the Gel Out gel extraction kit purchased from A\&A Biotechnology (Poland). The E. coli minipreps were performed using the Plasmid Mini Kit (A\&A Biotechnology). Transformation of $E$. coli strains was performed using standard chemical protocols [22]. Genomic DNA (gDNA) was extracted from Y. lipolytica using the Genomic Mini AX Yeast Spin kit (A\&A Biotechnology, Poland). The obtained plasmids were digested with MssI to create linear expression cassettes devoid of $E$. coli DNA and surrounded by $Y$. lipolytica rDNA for targeted integrations. The transformants were plated out on selective media [23] and were confirmed via gDNA extraction and three distinct PCR confirmations.

\section{Construction of overexpression plasmids}

First, plasmid pAD carrying UAS1B ${ }_{16}$-TEF promoter was formed. Primers pAD-NotI-F and pAD-NotI-R amplified linear plasmid pAD-UTGut1 [23] but devoid of the GUT1 gene. Next, the obtained PCR (7871 bp) product was digested with NotI and ligated, resulting in the pAD vector. The primers used in this study are listed in Additional file 1: Table S1.

After amplification of $Y$. lipolytica DNA with primers TKet-AscI-F and TKet-NheI-R, the 2458-bp PCR fragment was digested and cloned into the corresponding sites of pAD, resulting in pAD-TKL1. Gene $Y A L$ IOF15587g, encoding transaldolase, was amplified, by primers Tald-AscI-F and Tald-NheI-R. The 1283-bp PCR product was digested and cloned into the corresponding sites of pAD, resulting in pAD-TAL1.

Gene YALIOE22649g was amplified by primers ZWF1-AscI-F and ZWF1-PmlI-R, the 1957-bp PCR product was digested with $A s c \mathrm{I}(S g s \mathrm{I})$ and PmlI, and subsequently was cloned into the corresponding sites of pAD, yielding pAD-ZWF1.

Gene YALIOB15598g was amplified by primers GND1-AscI-F and GND1-NheI-R, the 2222-bp PCR product was digested with $A s c \mathrm{I}(S g s \mathrm{I})$ and NheI, and subsequently was cloned into the corresponding sites of pAD, yielding pAD-GND1. 


\section{Construction of hrGFP plasmids}

First, the gene hr-GFP from plasmid phrGFP II-C (Agilent) using primers hrGFP-AscI-F/hr-NheI-R was amplified, digested with the enzymes, and cloned into previously digested pAD, yielding pAD-hrGFP. Construction of the promoter-gfp fusion plasmids $\mathrm{P}_{\mathrm{TKL1}^{-}}$ gfp, $\mathrm{P}_{\mathrm{TAL}^{-}}$-gfp, $\mathrm{P}_{\mathrm{ZWF1}}$-gfp, $\mathrm{P}_{\mathrm{GND1}}$-gfp was carried out by amplifying the entire promoter region using primers Ptk-Bsp119I-F/Ptk-AscI-R, Ptal-Bsp119I-F/Ptal-AscIR/, Pzwf-Bsp119I-F/Pzwf-AscI-R, Pgnd-Bsp119I-F/ Pgnd-AscI-R. Subsequently, promoters were cloned into gel-extracted pAD (promotorless) digested with $A s c \mathrm{I} /$ Bsp119I, yielding $\mathrm{P}_{\mathrm{TKL}^{1}}$-gfp, $\mathrm{P}_{\mathrm{TAL1}^{-}}$-gfp, $\mathrm{P}_{\mathrm{ZWF}^{1}}$-gfp, $\mathrm{P}_{\mathrm{GND}^{-}}{ }^{-}$ gfp. The obtained plasmids were digested with MssI and transformed into the strain Y. lipolytica AMM.

\section{RNA isolation and transcript quantification}

The shake-flask cultures were grown for $24 \mathrm{~h}$ in Erythritol Synthesis Medium supplemented with glycerol $(100 \mathrm{~g} / \mathrm{L})$ or in the Control Medium supplemented with glucose $(100 \mathrm{~g} / \mathrm{L})$. Next, the cultures were collected and centrifuged for $5 \mathrm{~min}$ at $12,000 \mathrm{~g}$. The RNA was extracted using a Total RNA Mini Plus kit (A\&A Biotechnology, Poland). Each sample was treated with DNAse I (Thermo Scientific) according to the manufacturer's instructions. We measured RNA quantities using a Biochrom WPA Biowave II spectrophotometer (Biochrom Ltd., UK) equipped with a TrayCell (Hellma Analytics, Germany), and the samples were stored in a $-80{ }^{\circ} \mathrm{C}$ freezer. We conducted cDNA synthesis using Maxima First Strand cDNA. Synthesis kits for RTqPCR (Thermo Scientific) were used according to the manufacturer's instructions. We carried out qRT-PCR analyses using a DyNAmo Flash SYBR Green qPCR Kit (Thermo Scientific) and the Eco Real-Time PCR System (Illumina, USA). Primers for RT-PCR were designed as follows: gene TKL1 (YALIOEO6479g) encoding the transketolase, gene TAL1 (YALIOF15987g) encoding transaldolase, gene GND1 (YALIOB15598g) encoding $\mathrm{NADP}^{+}$-dependent 6-phosphogluconate dehydrogenase, gene ZWF1 (YALIOE22649g) encoding $\mathrm{NADP}^{+}$-dependent glucose-6-phosphate dehydrogenase were used as a template. Primers qTket-F and qTket- $\mathrm{R}$ bind to the first exon at $25 \mathrm{bp}$ and to the second exon at $478 \mathrm{bp}$ of the TKL1 gene, respectively, resulting in a 111 bp qRT-PCR product. Next, primers qTald-F and qTald-R bind to the first exon at $42 \mathrm{bp}$ and to the second exon at $388 \mathrm{bp}$ of the TAL1 gene, respectively, resulting in a $107 \mathrm{bp}$ qRTPCR product. Gene GND1 possesses three introns; thus primer qGND1-F binds to the second exon at $257 \mathrm{bp}$, and primer qGND1-R binds to the second exon at $576 \mathrm{bp}$. The obtained PCR product in qRT-PCR is $105 \mathrm{bp}$. Primers qZWF1-F and qZWF1-R bind to the first exon at
$5 \mathrm{bp}$ and to the second exon at $595 \mathrm{bp}$ of the $Z W F 1$ gene, respectively, resulting in a $151 \mathrm{bp}$ qRT-PCR product. The results were normalized to the actin gene ACT-F/ACT-R and analyzed using the ddCT method [24]. Samples were analyzed in triplicate.

\section{Analytical methods}

Samples $(10 \mathrm{~mL})$ from the cultures were centrifuged (10 min; $4{ }^{\circ} \mathrm{C} ; 5500 \times g$ ), harvested by filtration on 0.45 $\mu \mathrm{m}$ pore membranes and washed twice with distilled water. The biomass was determined gravimetrically after drying at $105{ }^{\circ} \mathrm{C}$. The concentrations of the metabolites were determined using HPLC equipped with a HyperRez Carbohydrate $\mathrm{H}^{+}$Column (Thermo Scientific, Waltham, MA) coupled to a UV $(\lambda=210 \mathrm{~nm})$ (Dionex, Sunnyvale, USA) and a refractive index (RI) detector (Shodex, Ogimachi, Japan). The column was eluted with $25 \mathrm{mM}$ of trifluoroacetic acid (TFA) at $65^{\circ} \mathrm{C}$ and a flow rate of $0.6 \mathrm{~mL} /$ $\min$.

\section{Fluorescence microscopy}

Yeast cells were visualized using an Axio Scope A1 Zeiss microscope (Zeiss, Germany). A GFP filterset (Zeiss), operated at excitation of $470 / 40 \mathrm{~nm}$, and emission of $525 / 50 \mathrm{~nm}$, was used to detect green fluorescence. Microscopy data were stored using ZEN lite (Zeiss).

\section{Calculation of fermentation parameters}

To consider medium dilution due to the addition of $\mathrm{NaOH}$ required for $\mathrm{pH}$ control, the amounts of erythritol and byproducts in the culture broth were used to calculate the mass yield of erythritol $\left(Y_{\text {ERY }}\right)$, and the volumetric erythritol productivity $\left(Q_{\text {ERY }}\right)$. The mass yield of erythritol $\left(Y_{\mathrm{ERY}}\right)$ was expressed in $\mathrm{g} / \mathrm{g}$ from glycerol and was calculated by the equation

$$
Y_{\mathrm{ERY}}=P / S .
$$

The volumetric erythritol productivity $\left(Q_{\mathrm{ERY}}\right)$ expressed in $\mathrm{g} / \mathrm{L} / \mathrm{h}$ was calculated from

$$
Q_{\text {ERY }}=P / V \cdot t \text {, }
$$

where $P$ is the amount of erythritol in the culture liquid at the end of cultivation (g); $S$ is the total amount of glycerol consumed (g); $V$ is the initial volume of culture liquid (l); and $\mathrm{t}$ is the fermentation time (h).

\section{Results and discussion}

\section{Quantification of gene expression during erythritol} synthesis

Production of erythritol by yeast is a well-known phenomenon; however, the metabolic pathway of this process for Y. lipolytica has never been characterized. Our previous study showed the hypothetical pathway of 
erythritol from glycerol in this oleaginous yeast [21]. In this study, we focused on four genes to investigate their role in erythritol biosynthesis. Here, we choose genes belonging to the pentose phosphate pathway (PPP), since it was shown that erythritol synthesis occurs via this pathway [25]. In this study, we selected genes encoding transketolase (TKL1, YALIOE06479g), transaldolase (TAL1, YALIOF15587g), $\mathrm{NADP}^{+}$-dependent 6-phosphogluconate dehydrogenase (GND1, YALIOB15598g), and $\mathrm{NADP}^{+}$-dependent glucose-6-phosphate dehydrogenase (ZWF1, YALIOE22649g). The first (oxidative) phase of PPP starts with dehydrogenation of glucose 6-phosphate, by 6-phosphogluconate dehydrogenase, resulting in NADPH and 6-phosphoglucono- $\delta$-lactone synthesis (Fig. 1). Next, 6-phosphoglucono- $\delta$-lactone is hydrolyzed by a specific lactonase to produce 6 -phosphogluconate. Consequently, this sugar is oxidatively decarboxylated by 6-phosphogluconate dehydrogenase, yielding ribulose-5-phosphate along with cogeneration of NADPH. In the second, nonoxidative phase of PPP, transketolase converts ribose-phosphate and xylose-5-phosphate into glyceraldehyde 3-phosphate (GAP) and sedoheptulose 7-phosphate. Next, these compounds are catalyzed by transaldolase to form fructose 6-phosphate and erythrose 4-phosphate. Transketolase converts glyceraldehyde3-phosphate with fructose-6-phosphate into xylulose5-phosphate and erythrose-4-phosphate (E4P). The last step of erythritol synthesis is beyond PPP, and it is a reduction of erythrose to erythritol by erythrose reductase with codominant NADPH.

The standard carbon source for yeast $Y$. lipolytica is glucose, and it is commonly used in research and in industry. However, during our study, we observed that the level of erythritol synthesis by strain Y. lipolytica MK1 depends on the carbon source, and it is higher when glycerol is applied (Additional file 2). Probably it is due to higher osmotic stress caused by glycerol. Moreover, in agreement with another study, we observed that in the medium containing glucose as a carbon source, strain MK1 forms mycelium [26]. In medium with glycerol, only yeast cells were observed; this suggests that mycelia cannot synthesize erythritol efficiently. Therefore, to verify the activity of the PPP genes during erythritol synthesis, we compared the relative expressions of the mentioned

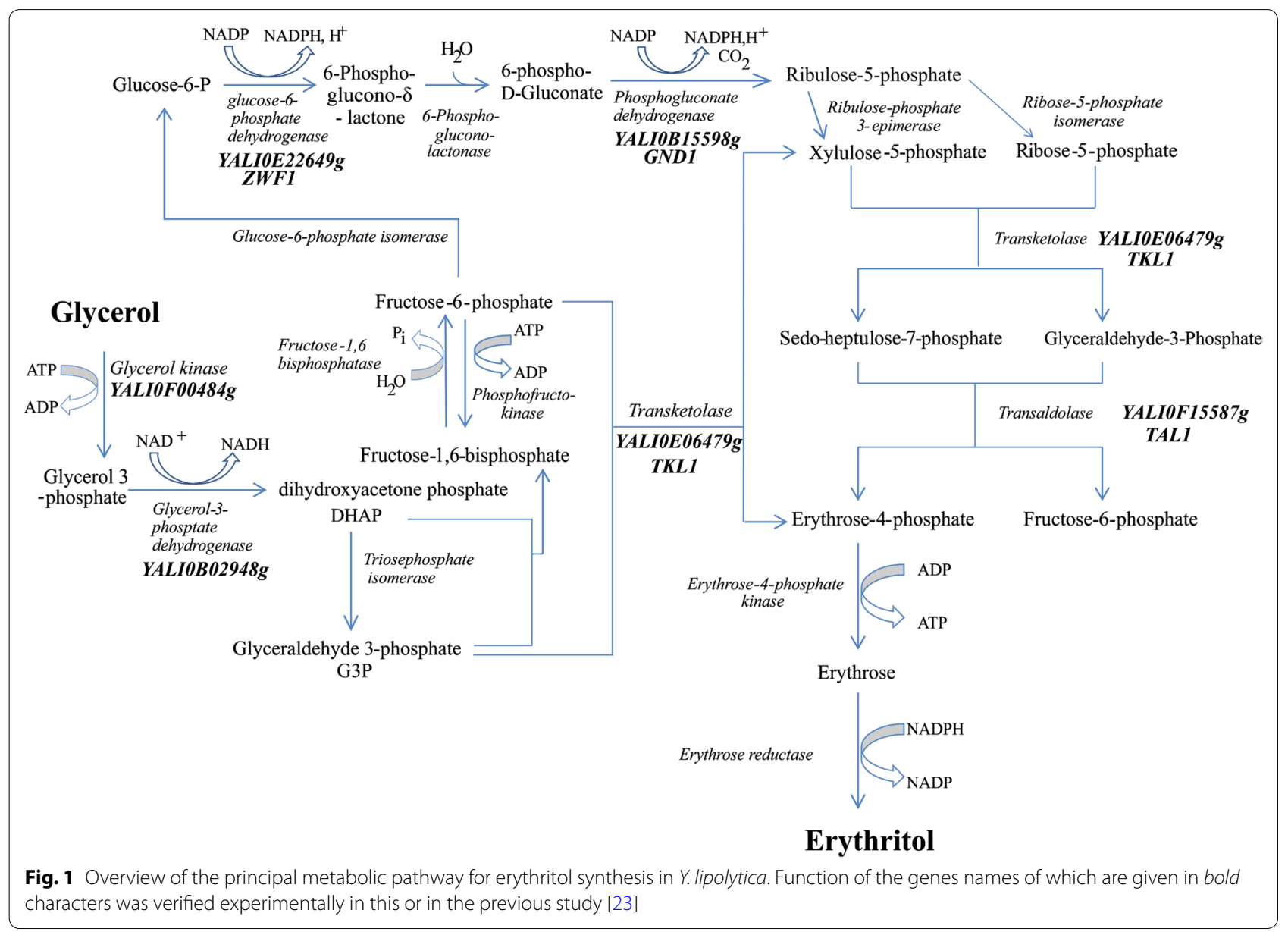


genes under two different conditions. First, the strain MK1 was grown in the Erythritol Synthesis Medium (see "Methods") in the baffled flasks; second, MK1 strain was grown in the Control Medium. Remarkably, immediately after $24 \mathrm{~h}$, gene TKL1 exhibited a sevenfold increase in expression over the control, and we also observed enhanced expressions for $Z W F 1$ and TAL1, by 3.0- and 1.8-fold respectively (Additional File 3 : Figure $\mathrm{S} 1$ ).

Interestingly, the expressions of the GND1 gene remain at the same level under both conditions (Fig. 2a). To confirm the qRT-PCR results, we fused the promoters of the mentioned genes with hrGFP protein, to visualize their activities under both conditions. In agreement with previous experiment, we observed enhanced GFP expressions for TLK1, ZWF1, and TAL1 in Erythritol Synthesis Medium (Fig. 2b). Strain AMM $\mathrm{P}_{\mathrm{GND1}}-g f p$ showed similar fluorescence under both conditions, suggesting that this dehydrogenase is active, independent of the applied carbon source. It is worth noting that we did not observe mycelium in the AMM-derived strains.

Previously, the activities of the enzymes encoded by these genes were studied for Moniliella megachiliensis (formerly Trichosporonoides megachiliensis) [20]. In that study, all enzymes belonging to PPP showed high activity during erythritol synthesis; however, transketolase was found as a crucial enzyme for the process. The results obtained in our study confirm that in Y. lipolytica, transketolase is expressed at an enhanced level in comparison with the other genes in the PPP.

\section{Overexpression of PPP genes in Y. lipolytica}

First, we sought to verify the influence of the PPP genes' overexpression on $Y$. lipolytica growth. Thus, we constructed overexpression cassettes containing the genes $T K L 1, T A L 1, Z W F 1$, and GND1 under a hybrid TEF promoter [27], and transformed the cassettes into the AMM strain, derived from MK1 [28], resulting in strains listed in Table 1.

Next, we monitored the growth of the engineered strains and the wild type in YNB media supplemented with glucose or glycerol. As seen in Fig. 3, all the engineered strains grew better on medium supplemented with glycerol than the control. In the first $24 \mathrm{~h}$, the growth of the all strains was similar; however, after this period, when the maximum activity of the TEF promoter started, we noted significant enhancement in growth of the engineered strains. The control strain grew on YNB with glycerol in the similar way as in the previous study [11]. We did not observe any significant differences in growth between the engineered strains, either on YNB with glucose (data not shown) or on YNB with glycerol.

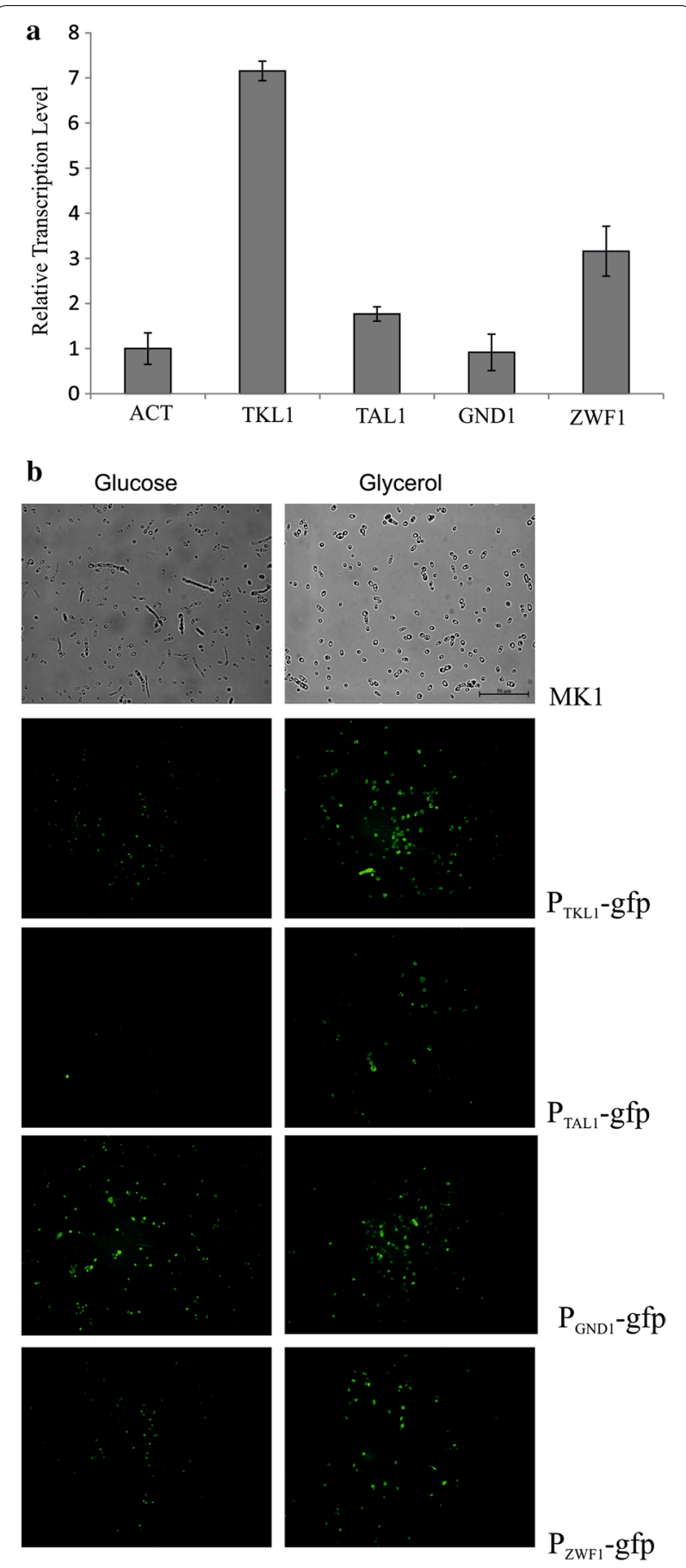

Fig. 2 a Expression of genes in this study. Relative quantification of RNA transcript using RT-PCR; actin was used as a reference gene. Strain MK1 was grown in Erythritol Synthesis Medium or in the Control Medium. Samples were analyzed in triplicate, and the standard errors were estimated using Illumina Eco software. $\mathbf{b}$ The visualization of the $P_{\text {TKL1 }}$-gfp, $P_{\text {TAL1 } 1}$-gfp, $P_{\text {GDN1 } 1}$-gfp, and $P_{Z W F 1}$-gfp expressions in $Y$. lipolytica AMM; strain MK1 was used as a control. Strains were grown in the Control Medium (left panels) or in the Erythritol Synthesis Medium (rights pane/s). Pictures were taken at $72 \mathrm{~h}$ of the cultivation. DIC pictures are shown in Additional File 4: Figure S2 


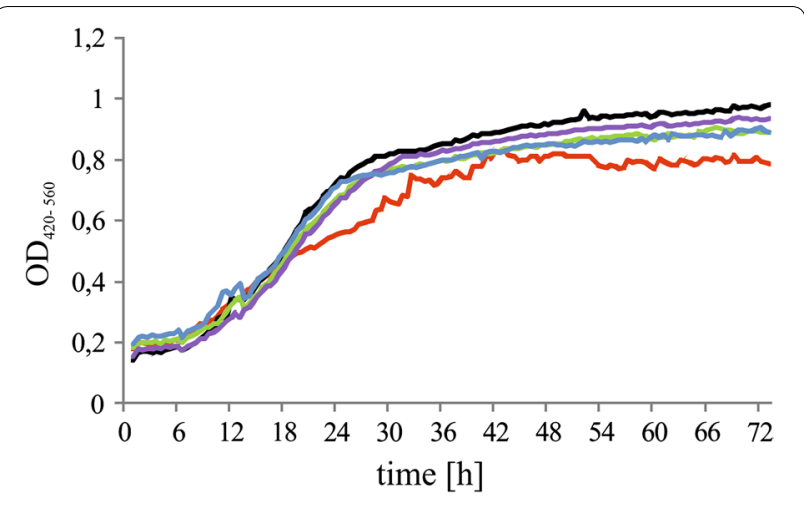

Fig. 3 Growth curves of various Y. lipolytica strains: MK1 (red), AMM pAD-TKL1 (black), AMM pAD-TAL1 (green), AMM pAD-GND1 (purple), and AMM pAD-ZWF1 (blue). The strains were grown on a YNB/glycerol medium. Quintuple experiments were performed at $28^{\circ} \mathrm{C}$ under constant agitation using Bioscreen $\mathrm{C}$
Taking into account this result, the effects of TKL1, $T A L 1, Z W F 1$, and GND1 overexpressions on erythritol synthesis were first assessed in the shake-flask experiment (Erlenmeyer flask). The conditions of the experiment were established previously [21]. In this study, we monitored two parameters: glycerol consumption and erythritol synthesis (Fig. 4a, b). After $24 \mathrm{~h}$, we did not observe any significant differences in substrate assimilation or in erythritol biosynthesis. However, after $48 \mathrm{~h}$, all the engineered strains showed elevated erythritol synthesis coupled with more rapid glycerol utilization. The most conspicuous effect was observed for the strain overexpressing the TKL1 gene; this strain produced $51.09 \pm 1.97 \mathrm{~g} / \mathrm{L}$ of erythritol within $94 \mathrm{~h}$. Erythritol titers for strains AMM pAD-TAL1, AMM pAD-ZWF1, and AMM pAD-GND1 were $46.69 \pm 1.59,42.53 \pm 2.62$, and $40.16 \pm 2.15 \mathrm{~g} / \mathrm{L}$, respectively. The control strain
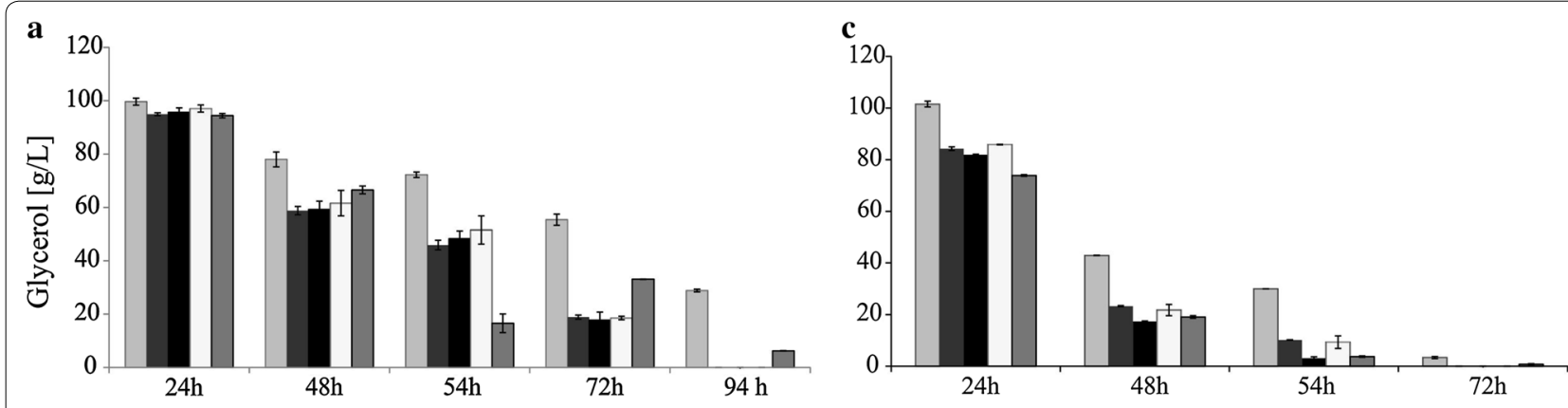

b
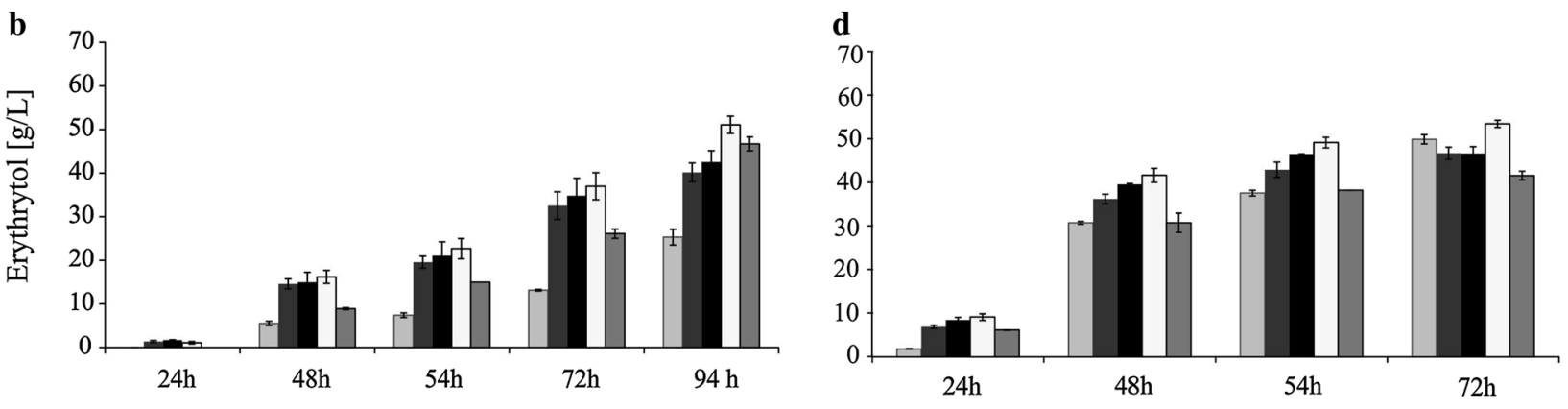

$\square \mathrm{MK} 1=\mathrm{pAD}-\mathrm{GND} 1$

-pAD-ZWF1

$\square$ pAD-TKL1 $\square$ pAD-TAL1

\begin{tabular}{lccc}
\hline Strain & $\begin{array}{c}\text { Erytrhitol } \\
{[\mathbf{g} / \mathbf{L}]}\end{array}$ & $\begin{array}{c}\mathbf{Q}_{\text {ERY }} \\
{[\mathbf{g} / \mathbf{L} \mathbf{h}]}\end{array}$ & $\begin{array}{c}\mathbf{Y}_{\text {ERY }} \\
{[\mathbf{g} / \mathbf{g}]}\end{array}$ \\
\hline Erlenmever flask & & & \\
MK1 & $25.30 \pm 1.83$ & 0.27 & 0.25 \\
AMM pAD-GND1 & $40.16 \pm 2.15$ & 0.43 & 0.40 \\
AMM pAD-ZWF1 & $42.53 \pm 2.62$ & 0.45 & 0.43 \\
AMM pAD-TLK1 & $51.09 \pm 1.97$ & 0.54 & 0.51 \\
AMM pAD-TAL1 & $46.69 \pm 1.59$ & 0.50 & 0.47 \\
\hline
\end{tabular}

\begin{tabular}{lccc}
\hline Strain & $\begin{array}{c}\text { Erytrhitol } \\
{[\mathbf{g} / \mathbf{L} \mathbf{l}}\end{array}$ & $\begin{array}{c}\mathbf{Q}_{\text {ERY }} \\
{[\mathbf{g} / \mathbf{L} \mathbf{~ h} \mathbf{l}}\end{array}$ & $\begin{array}{c}\mathbf{Y}_{\mathbf{E R Y}} \\
{[\mathbf{g} / \mathbf{g} \mathbf{l}}\end{array}$ \\
\hline Baffled flasks & & & \\
MK1 & $54.15 \pm 1.16$ & 0.75 & 0.54 \\
AMM pAD-GND1 & $50.67 \pm 1.52$ & 0.70 & 0.51 \\
AMM pAD-ZWF1 & $50.59 \pm 1.72$ & 0.70 & 0.51 \\
AMM pAD-TLK1 & $57.99 \pm 0.87$ & 0.81 & 0.58 \\
AMM pAD-TAL1 & $45.13 \pm 1.09$ & 0.63 & 0.45 \\
\hline
\end{tabular}

Fig. 4 Results of the shake-flask experiment in the Erlenmeyer flasks $(\mathbf{a}, \mathbf{b})$ and in the baffled flasks $(\mathbf{c}, \mathbf{d})$. Parameters of erythritol synthesis by various Y. lipolytica strains in shake-flask experiments at the end of the process; $Q_{E R Y}$ erythritol productivity; $Y_{\text {ERY }}$ erythritol yield. The cultures were performed in three biological replicates. The error bars represent the standard deviation. Tables summarize the parameters of erythritol synthesis at the end of the processes 
produced only $25.30 \pm 1.83 \mathrm{~g} / \mathrm{L}$. Although glycerol incorporation into the cell was not modified, we observed enhancement of glycerol utilization. In the cultures containing the overexpressing TKL1, ZWF1, and GND1 strains, after $94 \mathrm{~h}$, glycerol was totally depleted, whereas in the control strain, $28 \mathrm{~g} / \mathrm{L}$ of glycerol was detected. This phenomenon might be a result of pulling the metabolism toward erythritol synthesis by overexpression of the genes involved in this pathway. This has an enormous impact on the erythritol productivity $\left(Q_{E R Y}\right)$; the engineered strains achieved twofold higher productivity over the control strain. In addition, the yield of erythritol $\left(Y_{\text {ERY }}\right)$ was significantly improved (Fig. 4). The best impact on $Y_{\text {ERY }}$ was noted for strain AMM pAD-TKL1, where the yield achieved was $0.51 \mathrm{~g} / \mathrm{g}$, whereas for the control strain, $Y_{\text {ERY }}$ was only $0.35 \mathrm{~g} / \mathrm{g}$. It is worth noting that all the engineered strains showed enhanced capacity for erythritol biosynthesis from glycerol. Production of byproducts was also increased in the engineered strains; particularly, productions of mannitol and citric acid were significantly enhanced (Table 3 ). This is an effect of higher content of fructose 6-phosphate (in the PPP), which is a substrate for mannitol-1-phosphate and consequently for mannitol. The highest level of citric acid production was achieved by AMM pAD-GND1; this strain produced $18.54 \pm 1.67 \mathrm{~g} / \mathrm{L}$ of citric acid, which was threefold higher than that of the control strain $(5.98 \pm 0.10 \mathrm{~g} / \mathrm{L})$. Moreover, an enhanced level of citric acid is also caused by increased content of fructose 6-phosphate, which is one of the links of the chain in the Embden-Meyerhof-Parnas glycolytic pathway (EMP). The EMP is connected by pyruvate with the tricarboxylic acid (TCA) cycle. Therefore, overexpression of genes belonging to the PPP indirectly affect TCA.

It has been shown that oxygen concentration in the medium has a huge impact on erythritol synthesis by $M$. megachiliensis [20]. Therefore, to further characterize the engineered strains and explore their abilities of erythritol synthesis, the shake-baffled flask fermentation was conducted. Again, we monitored glycerol assimilation and erythritol synthesis (Fig. 4c, d). In agreement with our assumption, the process was much more efficient than when conducted in Erlenmeyer flasks. First, erythritol production started already after a few hours; therefore, at $24 \mathrm{~h}$ of growth, this polyol was detected for all tested strains, including the control. Total time of fermentation

Table 3 Side-metabolites of $Y$. lipolytica strains in shake-flask experiments in Erlenmeyer flasks

\begin{tabular}{|c|c|c|c|c|c|}
\hline Time (h) & Strain & Arabitol (g/L) & Mannitol (g/L) & Citric acid (g/L) & a-Ketoglutaric acid (g/L) \\
\hline \multirow[t]{5}{*}{24} & MK1 & 0 & 0 & 0 & 0 \\
\hline & AMM pAD-GND1 & $0.20 \pm 0.01$ & $0.05 \pm 0.01$ & $1.13 \pm 0.01$ & 0 \\
\hline & AMM pAD-ZWF1 & $0.24 \pm 0.03$ & $0.01 \pm 0.01$ & $0.78 \pm 0.01$ & 0 \\
\hline & AMM pAD-TKL1 & 0 & 0 & $0.84 \pm 0.01$ & 0 \\
\hline & AMM PAD-TAL 1 & 0 & $1.12 \pm 0.03$ & 0 & 0 \\
\hline \multirow[t]{5}{*}{48} & MK1 & $0.40 \pm 0.05$ & $0.32 \pm 0.06$ & $2.60 \pm 0.13$ & $0.55 \pm 0.02$ \\
\hline & AMM pAD-GND1 & $0.93 \pm 0.05$ & $2.15 \pm 0.02$ & $9.94 \pm 0.11$ & $0.44 \pm 0.05$ \\
\hline & AMM pAD-ZWF1 & $1.33 \pm 0.10$ & $1.99 \pm 0.23$ & $8.58 \pm 1.33$ & $0.53 \pm 0.08$ \\
\hline & AMM pAD-TKL1 & $0.87 \pm 0.10$ & $1.52 \pm 0.07$ & $6.32 \pm 2.18$ & $0.77 \pm 0.19$ \\
\hline & AMM PAD-TAL 1 & $1.04 \pm 0.07$ & $1.12 \pm 0.03$ & $5.05 \pm 1.27$ & $0.41 \pm 0.04$ \\
\hline \multirow[t]{5}{*}{54} & MK1 & $0.43 \pm 0.13$ & $0.39 \pm 0.09$ & $3.05 \pm 0.01$ & $0.66 \pm 0.06$ \\
\hline & AMM pAD-GND1 & $1.12 \pm 0.01$ & $3.00 \pm 0.09$ & $11.50 \pm 0.25$ & $0.45 \pm 0.20$ \\
\hline & AMM pAD-ZWF1 & $1.58 \pm 0.10$ & $2.82 \pm 0.15$ & $10.33 \pm 1.51$ & $0.68 \pm 0.15$ \\
\hline & AMM pAD-TKL1 & $1.09 \pm 0.01$ & $2.48 \pm 0.02$ & $7.64 \pm 2.51$ & $1.11 \pm 0.38$ \\
\hline & AMM PAD-TAL1 & $1.52 \pm 0.11$ & $2.11 \pm 0.45$ & $5.85 \pm 1.05$ & $0.66 \pm 0.13$ \\
\hline \multirow[t]{5}{*}{72} & MK1 & $0.64 \pm 0.01$ & $0.85 \pm 0.04$ & $4.54 \pm 0.14$ & $0.75 \pm 0.05$ \\
\hline & AMM pAD-GND1 & $1.32 \pm 0.12$ & $5.45 \pm 0.22$ & $16.24 \pm 0.71$ & $0.64 \pm 0.30$ \\
\hline & AMM pAD-ZWF1 & $2.01 \pm 0.20$ & $5.07 \pm 0.01$ & $13.62 \pm 3.31$ & $0.84 \pm 0.32$ \\
\hline & AMM pAD-TKL1 & $1.35 \pm 0.05$ & $4.41 \pm 0.05$ & $10.33 \pm 2.82$ & $1.60 \pm 0.61$ \\
\hline & AMM pAD-TAL1 & $1.70 \pm 0.16$ & $3.11 \pm 0.65$ & $7.92 \pm 0.69$ & $0.76 \pm 0.1$ \\
\hline \multirow[t]{5}{*}{94} & MK1 & $0.89 \pm 0.04$ & $1.71 \pm 0.16$ & $5.98 \pm 0.10$ & $0.79 \pm 0.08$ \\
\hline & AMM pAD-GND1 & $1.79 \pm 0.12$ & $7.63 \pm 0.48$ & $18.54 \pm 1.67$ & 0 \\
\hline & AMM pAD-ZWF1 & $2.61 \pm 0.16$ & $7.02 \pm 0.38$ & $15.32 \pm 4.28$ & 0 \\
\hline & AMM pAD-TKL1 & $1.53 \pm 0.39$ & $6.66 \pm 0.78$ & $12.70 \pm 2.18$ & $0.31 \pm 0.10$ \\
\hline & AMM PAD-TAL1 & $2.30 \pm 0.13$ & $6.23 \pm 0.36$ & $11.16 \pm 1.41$ & $1.14 \pm 0.52$ \\
\hline
\end{tabular}


was only $72 \mathrm{~h}$ for all the engineered strains; for the control, residual glycerol was still detected $(3.34 \mathrm{~g} / \mathrm{L})$. Increased aeration has the highest influence on the MK1 strain, because the titer of erythritol at the end of the process $(51.15 \pm 1.16 \mathrm{~g} / \mathrm{L})$ increased twofold in comparison with the previous experiment $(25.3 \pm 1.83 \mathrm{~g} / \mathrm{L})$. Nevertheless, changes of the condition also improved production of the $T K L 1$ overexpressing strain. Here, the erythritol titer was the highest among the tested strains, and it achieved $58.0 \pm 0.87 \mathrm{~g} / \mathrm{L}$. This leads to enhancement of the process parameters; $Y_{\mathrm{ERY}}$ achieved $0.58 \mathrm{~g} / \mathrm{g}$ and $Q_{\text {ERY }} 0.81 \mathrm{~g} / \mathrm{L} \mathrm{h}$. This is one of the highest amounts of erythritol titers obtained in the shake-flask experiments. A previously conducted experiment for $Y$. lipolytica in a shake-flask experiment showed a lower titer levels (20.03-35.53 g/L), $Y_{\text {ERY }}(0.33-0.42 \mathrm{~g} / \mathrm{g})$, and $Q_{\text {ERY }}(0.08-$ $0.15 \mathrm{~g} / \mathrm{L} \mathrm{h}$ ) [29]. Also in experiments using Torula sp. supplementation of culture media resulted in enhanced amount of erythritol titer, which was $56.6 \mathrm{~g} / \mathrm{L}$, whereas $Y_{\text {ERY }}$ achieved was only $0.28 \mathrm{~g} / \mathrm{g}$ [30].

Surprisingly, overexpressions of ZWF1 and GND1 at higher aeration resulted in higher production of erythritol than at low aeration (Fig. 4); however, after glycerol depletion, we noted rapid polyol assimilation. As a result, both stains showed a slightly lower erythritol titer than the control at the end of the process. These data suggest that both dehydrogenases might play a role in erythritol assimilation as a potential carbon source.
Next, strain AMM pAD-TAL1 under high aeration conditions produced less erythritol than in low aeration condition. However, we noted that carbon flux was pushed toward citric acid synthesis. This strain produced $23.37 \pm 0.92 \mathrm{~g} / \mathrm{L}$ of citric acid in baffled flasks, which was a twofold improvement compared with Erlenmeyer flasks, where the citric acid level achieved was $11.16 \pm 1.41 \mathrm{~g} / \mathrm{L}$. Moreover, it was the highest titer of citric acid among the tested strains (Table 4). This undesired effect for the downstream process can be eliminated by optimization of the fermentation conditions. It is worth noting that the optimization of fermentation conditions is important and has the same high impact on the process as metabolic engineering. Therefore, all genetic modifications should be coupled with the optimization of the fermentation conditions. In accordance with the aim of this study, and to further characterize the AMM pAD-TKL1 transformant and explore its erythritol production characteristics, a large-scale experiment was performed using a 5-L bioreactor.

\section{The effect of TKL1 overexpression in bioreactor fermentations}

Given the results of the study, we sought to cultivate AMM pAD-TKL1 strain in a bioreactor fermentation, consistent with experiments previously conducted [23]. This strain was chosen since TKL1 was found to be a crucial gene for erythritol production, and a transformant

Table 4 Side-metabolites of $Y$. lipolytica strains in shake-flask experiments in baffled flasks

\begin{tabular}{|c|c|c|c|c|c|}
\hline Time (h) & Strain & Arabitol (g/L) & Mannitol (g/L) & Citric acid (g/L) & a-Ketoglutaric acid (g/L) \\
\hline \multirow[t]{5}{*}{24} & MK1 & $0.24 \pm 0.03$ & 0 & $2.38 \pm 0.03$ & $0.23 \pm 0.01$ \\
\hline & AMM pAD-GND1 & $0.15 \pm 0.04$ & $0.29 \pm 0.01$ & $8.05 \pm 0.05$ & $0.25 \pm 0.00$ \\
\hline & AMM pAD-ZWF1 & $0.35 \pm 0.01$ & $0.37 \pm 0.05$ & $9.00 \pm 1.30$ & $0.33 \pm 0.04$ \\
\hline & AMM pAD-TKL1 & $0.26 \pm 0.01$ & $0.32 \pm 0.03$ & $7.61 \pm 0.21$ & $0.47 \pm 0.02$ \\
\hline & AMM pAD-TAL1 & 0 & $3.20 \pm 0.05$ & $7.10 \pm 0.50$ & 0 \\
\hline \multirow[t]{5}{*}{48} & MK1 & $0.77 \pm 0.00$ & $1.19 \pm 0.01$ & $9.53 \pm 0.36$ & $0.44 \pm 0.01$ \\
\hline & AMM pAD-GND1 & $0.70 \pm 0.02$ & $3.14 \pm 0.02$ & $14.26 \pm 0.08$ & $1.12 \pm 0.08$ \\
\hline & AMM pAD-ZWF1 & $1.05 \pm 0.01$ & $3.22 \pm 0.13$ & $15.37 \pm 3.54$ & $1.31 \pm 0.24$ \\
\hline & AMM pAD-TKL1 & $0.52 \pm 0.00$ & $2.43 \pm 0.07$ & $13.64 \pm 0.54$ & $2.10 \pm 0.11$ \\
\hline & AMM pAD-TAL1 & $0.93 \pm 0.02$ & $3.20 \pm 0.05$ & $17.03 \pm 2.82$ & $0.27 \pm 0.19$ \\
\hline \multirow[t]{5}{*}{54} & MK1 & $0.76 \pm 0.05$ & $1.35 \pm 0.27$ & $9.99 \pm 0.28$ & $0.45 \pm 0.01$ \\
\hline & AMM pAD-GND1 & $0.74 \pm 0.02$ & $3.70 \pm 0.05$ & $15.60 \pm 0.24$ & $1.10 \pm 0.08$ \\
\hline & AMM pAD-ZWF1 & $1.08 \pm 0.02$ & $3.79 \pm 0.13$ & $16.64 \pm 4.04$ & $1.29 \pm 0.24$ \\
\hline & AMM pAD-TKL1 & $0.54 \pm 0.00$ & $2.87 \pm 0.05$ & $15.61 \pm 0.54$ & $2.04 \pm 0.13$ \\
\hline & AMM PAD-TAL1 & $1.16 \pm 0.07$ & $4.73 \pm 0.15$ & $13.67 \pm 1.77$ & $0.00 \pm 0.00$ \\
\hline \multirow[t]{5}{*}{72} & MK1 & $0.71 \pm 0.07$ & $1.90 \pm 0.08$ & $10.69 \pm 0.27$ & $0.37 \pm 0.02$ \\
\hline & AMM pAD-GND1 & $0.75 \pm 0.04$ & $4.26 \pm 0.10$ & $15.95 \pm 0.34$ & 0 \\
\hline & AMM pAD-ZWF1 & $1.08 \pm 0.10$ & $4.27 \pm 0.09$ & $15.97 \pm 3.83$ & 0 \\
\hline & AMM pAD-TKL1 & $0.55 \pm 0.01$ & $3.40 \pm 0.06$ & $17.35 \pm 1.60$ & 0 \\
\hline & AMM pAD-TAL1 & $0.96 \pm 0.25$ & $4.72 \pm 0.40$ & $23.37 \pm 0.92$ & 0 \\
\hline
\end{tabular}


overexpressing TKL1 showed the highest capacity for erythritol synthesis. During cultivation of Y. lipolytica on an industrial scale, one of the important issues is the high cost of energy consumption for continuous and intense agitation. This yeast involves high demand for oxygen; otherwise the fermentation parameters significantly decrease. In the shake-flask experiment, as we observed that low aeration did not have a negative influence on erythritol synthesis, we sought to conduct the fermentation process at lower agitation $(500 \mathrm{rpm})$ than that applied in the standard procedures $(800 \mathrm{rpm})$. The aeration was fixed at $0.8 \mathrm{~L} / \mathrm{min}$, and again as a control strain, MK1 was used.

In agreement with the other studies [31], the dissolved oxygen fell to below $1 \%$ after $24 \mathrm{~h}$ of growth. As seen in Fig. 5a for the strain overexpressing TKL1, low oxygen was not an obstacle to growth. At the end of the process, the biomass of AMM pAD-TKL1 strain reached $20 \mathrm{~g} / \mathrm{L}$, which is a standard biomass titer in the erythritol synthesis medium [23]. In contrast, the control strain produced a maximum $14.1 \mathrm{~g} / \mathrm{L}$ biomass after $96 \mathrm{~h}$ of growth, and it decreased to $10 \mathrm{~g} / \mathrm{L}$ at the end of the process. The first detection of erythritol was after $24 \mathrm{~h}$ for both stains, and we did not notice any difference between the two strains. However, after the next $24 \mathrm{~h}$, when the highest activity of the TEF promoter started, we observed rapid production of erythritol for the engineered strain (Fig. 5a). The engineered strain produced $62.5 \mathrm{~g} / \mathrm{L}$ of erythritol within $102 \mathrm{~h}$, resulting in $Q_{\text {ERY }} 0.62 \pm 0.05 \mathrm{~g} / \mathrm{L} \mathrm{h}$ and $Y_{\text {ERY }} 0.42 \pm 0.05 \mathrm{~g} / \mathrm{g}$. Interestingly, production of the side-metabolites was significantly inhibited in comparison with the shake-flask experiment, and the maximum content of each of the undesired products did not exceed $5 \mathrm{~g} / \mathrm{L}$ (Table 5 ). This effect was caused by the established and fully controlled fermentations process in the bioreactor; $\mathrm{pH}$ and the oxygen supply
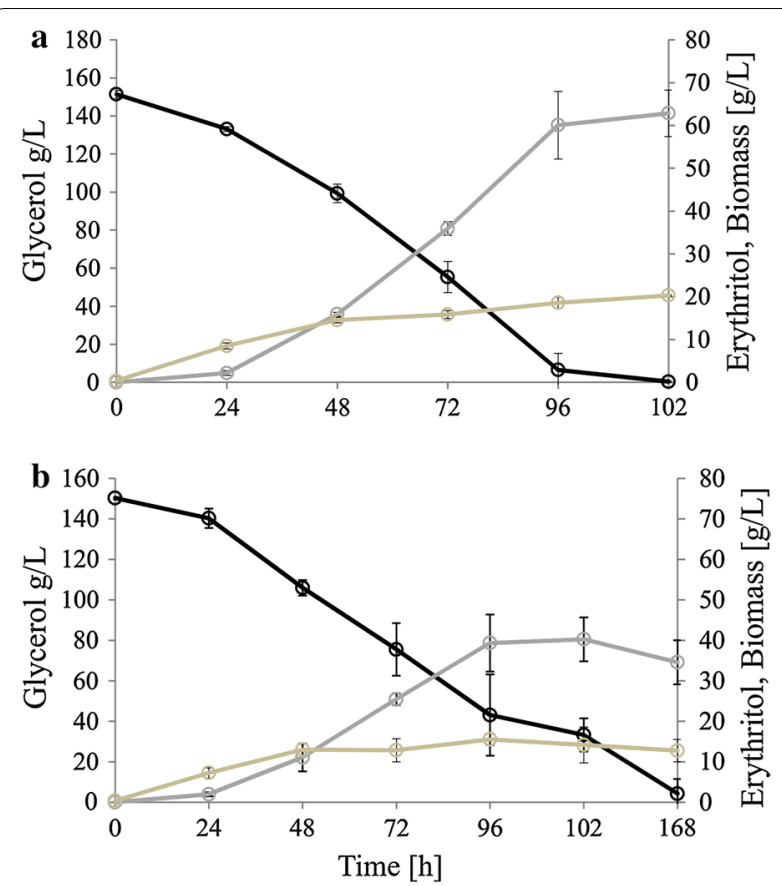

Fig. 5 Batch bioreactor fermentations with strain overexpressing TKL1 (a), and strain MK1 used as a control (b). Glycerol assimilation (black line), erythritol synthesis (gray line), and biomass production (brown line). The cultures were performed in three biological replicates. The error bars represent the standard deviation

were maintained at constant levels. A similar effect during erythritol production was observed previously [21].

The low concentration of side-metabolites is important for industrial applications, since it simplifies the purification process and consequently reduces production costs. The engineered strain assimilated glycerol within $102 \mathrm{~h}$ of fermentation, whereas the control strain was not able to deplete glycerol during $168 \mathrm{~h}$ of cultivation. Moreover, biosynthesis of erythritol was limited compare to

Table 5 Side-metabolites of $Y$. lipolytica strains in bioreactor study

\begin{tabular}{|c|c|c|c|c|c|}
\hline Time (h) & Strain & Arabitol (g/L) & Mannitol (g/L) & Citric acid (g/L) & a-Ketoglutaric acid (g/L) \\
\hline \multirow[t]{2}{*}{24} & MK1 & 0 & 0 & 0 & 0 \\
\hline & AMM pAD-TKL1 & 0 & 0 & $0.02 \pm 0.02$ & $0.25 \pm 0.07$ \\
\hline \multirow[t]{2}{*}{48} & MK1 & $0.35 \pm 0.07$ & 0 & $0.15 \pm 0.2$ & $0.70 \pm 0.14$ \\
\hline & AMM pAD-TKL1 & $0.76 \pm 0.08$ & 1.000 .01 & $0.14 \pm 0.10$ & $1.21 \pm 0.13$ \\
\hline \multirow[t]{2}{*}{72} & MK1 & $0.70 \pm 0.01$ & $0.35 \pm 0.19$ & $0.40 \pm 0.17$ & $0.90 \pm 0.14$ \\
\hline & AMM pAD-TKL1 & $1.21 \pm 0.16$ & 2.720 .03 & $0.93 \pm 0.52$ & $2.93 \pm 0.38$ \\
\hline \multirow[t]{2}{*}{96} & MK1 & $0.85 \pm 0.07$ & $1.20 \pm 0.42$ & $0.70 \pm 0.57$ & $0.90 \pm 0.42$ \\
\hline & AMM pAD-TKL1 & $1.33 \pm 0.46$ & 4.011 .42 & $1.63 \pm 0.67$ & $4.07 \pm 0.90$ \\
\hline \multirow[t]{2}{*}{102} & MK1 & $0.90 \pm 0.14$ & $1.15 \pm 0.49$ & $0.80 \pm 0.51$ & $0.95 \pm 0.49$ \\
\hline & AMM pAD-TKL1 & $1.40 \pm 0.57$ & 5.000 .28 & $4.00 \pm 1.97$ & $3.95 \pm 1.77$ \\
\hline \multirow[t]{2}{*}{168} & MK1 & $0.15 \pm 0.21$ & $0.25 \pm 0.20$ & $1.20 \pm 0.28$ & 0 \\
\hline & AMM pAD-TKL1 & - & - & - & - \\
\hline
\end{tabular}


the engineered strain, and the titer was $37.3 \mathrm{~g} / \mathrm{L}$ at the end of the process, resulting in $Q_{\mathrm{ERY}} 0.27 \mathrm{~g} / \mathrm{L} \mathrm{h}$ and $Y_{\mathrm{ERY}}$ $0.25 \mathrm{~g} / \mathrm{g}$. Due to weak growth of the control strain, production of byproducts was highly limited (Table 5).

The process conducted for the control strain at low agitation was inefficient and time consuming compares with the previous studies $[5,21,32]$. These data confirm that oxygen limitation has a huge impact on erythritol synthesis by $Y$. lipolytica. These results showed that transketolase plays a crucial role in erythritol synthesis and its overexpression allows for robust growth independent of oxygen supply. The engineered strain AMM PAD-TKL1 synthesized erythritol efficiently in a short period of time at a low oxygen concentration. It has a huge potential for industrial application.

\section{Conclusions}

Until now, the metabolic pathway of erythritol synthesis in Y. lipolytica has been never described. In this study, we showed that erythritol production in Y. lipolytica occurs via the pentose phosphate pathway (PPP) and the gene YALIOE06479g encoding transketolase plays a crucial role in this process. Moreover, gene YALIOE22649g encoding glucose-6-phosphate dehydrogenase (ZWF1) provides the reducing agent which is required in the last step of the process. Overexpression of the genes belonging to the PPP enhances the level of erythritol titer. An increased level of $T K L 1$ transcription results in significantly higher erythritol synthesis under conditions of oxygen limitation. During the shake-flask experiment, the engineered strain increased erythritol production twofold over the control. Over the course of the bioreactor study, strain AMM pAD-TKL1 produced $67 \%$ more erythritol than the control, and production of the by-product was significantly inhibited. Moreover, these results were obtained using a low-cost medium in which the single carbon source was glycerol and at low agitation; this situation is beneficial for downstream processing.

\section{Additional files}

Additional file 1: Table S1. The primer list used in this study.

Additional file 2. Production of erythritol and carbon source utilization in the baffled flasks conducted in the Erythritol Synthesis medium and in the Control Medium.

Additional file 3: Figure S1. Quantification of gene expression during erythritol synthesis by the strain MK1 strain at $24 \mathrm{~h}$ of growth in Erlenmeyer flasks. Samples were analyzed in triplicate and the standard errors were estimated using Illumina Eco software.

Additional file 4: Figure S2. The visualization of the PTKL1-gfp, PTAL1gfp, PGDN1-gfp and PZWF1-gfp expression in Y. lipolytica AMM. Strains were grown in the Control Medium (left panels) or in the Erythritol Synthesis Medium (rights panels). Pictures were taken at $72 \mathrm{~h}$ of the cultivation.

\section{Abbreviations}

TKL 1: gene YALIOE06479g encoding transketolase; TAL 1: gene YALIOF15587g encoding transaldolase; ZWF1: gene YALIOE22649g encoding glucose-6-phosphate dehydrogenase; GND1: gene YALIOB15598g encoding 6-phosphogluconate dehydrogenase.

\section{Authors' contributions}

AMM designed the study, constructed the plasmids and the strains, performed qRT-PCR study, took microscopy pictures, analyzed the data, and wrote the manuscript. $A B$ participated in the fermentation experiments. $A D$ participated in the design of the study, helped to analyze the data, and revised the manuscript. All authors read and approved the final manuscript.

\section{Acknowledgements}

The authors thank Magdalena Rakicka for HPLC analysis.

\section{Competing interests}

The authors declare that they have no competing interests.

Availability of supporting data

The authors promise the availability of supporting data.

\section{Consent for publication}

The authors have consented for publication.

\section{Funding}

This work was financed by the Polish National Centre for Research and Development under project LIDER/010/207/L-5/13/NCBR/2014.

Received: 19 January 2017 Accepted: 22 March 2017

Published online: 24 March 2017

\section{References}

1. Moon HJ. Biotechnological production of erythritol and its applications. Appl Microbiol Biotechnol. 2010;86:1017-25

2. Bernt WO, et al. Erythritol: a review of biological and toxicological studies. Regul Toxicol Pharmacol. 1996;24(2 Pt 2):S191-7.

3. Oh DK, et al. Increased erythritol production in fed-batch cultures of Torula sp. by controlling glucose concentration. J Ind Microbiol Biotechnol. 2001;26(4):248-52.

4. Segueilha L. Method producing erythritol by repeated fed-batch fermentation. In: Freres R, editor. 09/337909 United States Patent. 2002.

5. Rymowicz W, Rywinska A, Marcinkiewicz M. High-yield production of erythritol from raw glycerol in fed-batch cultures of Yarrowia lipolytica. Biotechnol Lett. 2009:31(3):377-80.

6. Mirończuk AM, et al. Enhanced production of erythritol by Yarrowia lipoIytica on glycerol in repeated batch cultures. J Ind Microbiol Biotechnol. 2014;41(1):57-64

7. Park SY, Seo JH, Ryu YW. Two-stage fed-batch culture of Candida magnoliae for the production of erythritol using an industrial medium. Korean J Biotechnol Bioeng. 2003;18(4):249-54.

8. Ryu YW, Park CY, Park JB, Kim SY, Seo JH. Optimization of erythritol production by Candida magnoliae in fed-batch culture. J Ind Microbiol Biotechnol. 2000;25(2):100-3.

9. Yang L-B. A novel osmotic pressure control fed-batch fermentation strategy for improvement of erythritol production by Yarrowia lipolytica from glycerol. Bioresour Technol. 2014;151:120-7.

10. Makri A. Metabolic activities of biotechnological interest in Yarrowia lipolytica grown on glycerol in repeated batch cultures. Bioresour Technol. 2010;101:2351-8.

11. Dobrowolski A. Efficient conversion of crude glycerol from various industrial wastes into single cell oil by yeast Yarrowia lipolytica. Bioresour Technol. 2016;207:237-43.

12. Kamzolova SV, et al. The effect of oxalic and itaconic acids on threoDs-isocitric acid production from rapeseed oil by Yarrowia lipolytica. Bioresour Technol. 2016;206:128-33. 
13. Lazar Z. Simultaneous production of citric acid and invertase by Yarrowia lipolytica SUC+ transformants. Bioresour Technol. 2011;102:6982-9.

14. Mirończuk A, Rywińska A, Rymowicz W. High-level production of erythritol by Yarrowia lipolytica MK1 from glycerol. Yeast. 2013;30(S1):97-216.

15. Otto $C$, et al. Variation of the by-product spectrum during alpha-ketoglutaric acid production from raw glycerol by overexpression of fumarase and pyruvate carboxylase genes in Yarrowia lipolytica. Appl Microbiol Biotechnol. 2012;95(4):905-17.

16. Rakicka M, et al. Technology of efficient continuous erythritol production from glycerol. J Clean Prod. 2016;139:905-13.

17. Rymowicz W, et al. Citric acid production from glycerol-containing waste of biodiesel industry by Yarrowia lipolytica in batch, repeated batch, and cell recycle regimes. Appl Microbiol Biotechnol. 2010;87(3):971-9.

18. Yovkova $V$, et al. Engineering the alpha-ketoglutarate overproduction from raw glycerol by overexpression of the genes encoding NADP(+)dependent isocitrate dehydrogenase and pyruvate carboxylase in Yarrowia lipolytica. Appl Microbiol Biotechnol. 2014;98(5):2003-13.

19. Lee $\mathrm{DH}$, et al. Molecular cloning and biochemical characterization of a novel erythrose reductase from Candida magnoliae JH110. Microb Cell Fact. 2010;9:43.

20. Sawada K, et al. Key role for transketolase activity in erythritol production by Trichosporonoides megachiliensis SN-G42. J Biosci Bioeng. 2009;108(5):385-90.

21. Mirończuk AM, et al. Newly isolated mutant of Yarrowia lipolytica MK1 as a proper host for efficient erythritol biosynthesis from glycerol. Process Biochem. 2015;50(1):61-8.

22. Sambrook J. Molecular cloning: a laboratory manual. 3rd ed. New York: Cold Spring Harbor Laboratory Press; 2001.
23. Mirończuk AM, et al. A novel strain of Yarrowia lipolytica as a platform for value-added product synthesis from glycerol. Biotechnol Biofuels. 2016;9(1):1-12.

24. Schmittgen TD, Livak KJ. Analyzing real-time PCR data by the comparative C(T) method. Nat Protoc. 2008;3(6):1101-8.

25. Moon HJ, et al. Biotechnological production of erythritol and its applications. Appl Microbiol Biotechnol. 2010;86(4):1017-25.

26. Ruiz-Herrera J, Sentandreu R. Different effectors of dimorphism in Yarrowia lipolytica. Arch Microbiol. 2002;178(6):477-83.

27. Blazeck J, et al. Tuning gene expression in Yarrowia lipolytica by a hybrid promoter approach. Appl Environ Microbiol. 2011;77(22):7905-14.

28. Mirończuk AM, et al. A two-stage fermentation process of erythritol production by yeast $Y$. lipolytica from molasses and glycerol. Bioresour Technol. 2015;198:445-55.

29. Tomaszewska L, Rywinska A, Gladkowski W. Production of erythritol and mannitol by Yarrowia lipolytica yeast in media containing glycerol. J Ind Microbiol Biotechnol. 2012;39(9):1333-43.

30. Kim KA, et al. Optimization of culture conditions for erythritol production by Torula sp. Seoul: Korean Society for Applied Microbiology; 2000.

31. Jost $B$, et al. The influence of oxygen limitation for the production of succinic acid with recombinant strains of Yarrowia lipolytica. Appl Microbiol Biotechnol. 2015:99(4):1675-86.

32. Rakicka M, et al. Enhanced production of erythritol and mannitol by Yarrowia lipolytica in media containing surfactants. Braz J Microbiol. 2016;47(2):417-23.

33. Hanahan D. DNA cloning: a practical approach. In: Glover DM, editor. McLean: IRL Press; 1985.

\section{Submit your next manuscript to BioMed Central and we will help you at every step:}

- We accept pre-submission inquiries

- Our selector tool helps you to find the most relevant journal

- We provide round the clock customer support

- Convenient online submission

- Thorough peer review

- Inclusion in PubMed and all major indexing services

- Maximum visibility for your research

Submit your manuscript at www.biomedcentral.com/submit
O Biomed Central 